\title{
Dinámica anual de tintínidos en las aguas estuarinas de dos áreas marinas de pesca responsable en el Golfo de Nicoya, Costa Rica
}

\author{
Tintinnids Diversity and Abundance in Estuarine Waters of Two Marine Areas for \\ Responsible Fishing in the Gulf of Nicoya, Costa Rica
}

Andrea García-Rojas

andrea.garcia.rojas@una.cr

Estación de Biología Marina Juan Bertoglia Richards

Escuela de Ciencias Biológicas, Universidad Nacional

Puntarenas, Costa Rica

Hannia Vega-Bolaños

hannia.vega.bolanos@una.cr

Estación de Biología Marina Juan Bertoglia Richards

Escuela de Ciencias Biológicas, Universidad Nacional

Puntarenas, Costa Rica

Valeria Quesada-Phillips

valeqp11@gmail.com

Estación de Biología Marina Juan Bertoglia Richards

Escuela de Ciencias Biológicas, Universidad Nacional

Puntarenas, Costa Rica

Recibido-Received: 10/dic/2015 / Corregido-Corrected: 13/jun /2016.

Aceptado-Accepted: 20/ago/2016 / Publicado-Published: 31/ene /2017.

\begin{abstract}
Resumen
En los periodos de marzo de 2012 a marzo de 2013, y de setiembre del 2013 a agosto del 2014, se determinó la biodiversidad y abundancia de tintínidos y su relación con variables ambientales, en dos áreas marinas de pesca responsable (AMPR) (Puerto Níspero y Paquera) del Golfo de Nicoya, Costa Rica. Para ambas zonas de muestreo se determinó una estacionalidad de los parámetros físico-químicos influenciada por variabilidad climática de la zona, lo cual se evidenció con las diferencias significativas en el tiempo de muestreo. La población de tintínidos reflejó una baja diversidad de especies donde los principales representantes fueron Tintinnopsis parva, T. uruguayensis y Tintinnidium balechi; y su abundancia osciló entre un rango de 0 a 5583 cél $\mathrm{L}^{-1}$. Al correlacionar la abundancia de tintínidos con respecto a las variables ambientales estudiadas en cada sitio de muestreo, se determinó una tendencia a que las interacciones significativas fueran negativas, principalmente entre la abundancia de tintínidos y la concentración de oxígeno disuelto en ambas AMPR. También, al interrelacionar las variables ambientales respecto al comportamiento temporal de la abundancia de tintínidos, se determinó que sí hay una relación entre el aumento en la concentración de los organismos y la variación ambiental. A nivel general, la presencia de tintínidos evidencia el proceso natural de la cadena trófica, al garantizar una dinámica productiva en la base de la cadena alimenticia de las zonas de estudio.
\end{abstract}

Palabras claves: Tintínidos; plancton; biodiversidad; estuario. 
UNICIENCIA Vol. 31, No. 1, pp. 1-12. Enero-junio, 2017.

ISSN Electrónico: 2215-3470

URL: www.revistas.una.ac.cr/uniciencia

DOI: http://dx.doi.org/10.15359/ru.31-1.1

Email: revistauniciencia@una.cr

\begin{abstract}
From March 2012 to March 2013, and from September 2013 to August 2014, biodiversity and abundance of tintinnids and its relationship with environmental variables were determined in two Marine Areas for Responsible Fisheries (AMPR) (Puerto Níspero and Paquera) of Gulf of Nicoya, Costa Rica. For both sampling areas, it was determined seasonality of the physicochemical parameters influenced by the climate variability of the zone, which was evidenced by the significant differences in the sampling time. Tintinnids population showed a low diversity of species where the main representatives were Tintinnopsis parva, T. uruguayensis, and Tintinnidium balechi; and their abundance ranged from a 0 to 5583 cells $\mathrm{L}^{-1}$. By correlating the abundance of tintinnids regarding environmental variables studied in each sampling site, a tendency for significant interactions were negative, mainly tintinnids abundance and concentration of dissolved oxygen in both AMPR. Also, environmental variables interrelate with respect to temporal behavior of the abundance of tintinnids; it was determined whether there is a relationship between the increase in the concentration of organisms and environmental variation. Overall, the presence of tintinnids shows the natural process of the trophic web, ensuring a productive dynamics at the base of the food chain of the study areas.
\end{abstract}

Keywords: tintinnids; plankton; biodiversity; estuary.

En el Golfo de Nicoya, la implementación de áreas marinas de pesca responsable (AMPR) surge como una medida de compromiso de las comunidades costeras con el manejo sostenible de las pesquerías, y ha representado una estrategia fundamental en la ordenación de las aguas marino-costeras del golfo. Para la ratificación de una zona como AMPR, son fundamentales los estudios de línea base donde se complementen la información pesquera con la descripción e identificación de las características propias del ecosistema, por lo cual, la determinación de los diferentes eslabones de la cadena trófica es indispensable.

Uno de los principales componentes de la estructura trófica marina es el zooplancton, dentro del cual los tintínidos representan uno de los principales componentes del microzooplancton, y pueden llegar a ser una población relevante en aguas estuarinas y salobres (Abou Zaid \& Hellal, 2012; Alder, 1999; Moscatello, Caroppo, Hajdëri \& Belmonte, 2011; Rakshit, Ganesh \& Kumar Sarkar, 2015b; Santoferrara \& Alder, 2012; Thompson, Alder \& Boltovskoy, 2001; Yu et al., 2015). Estos organismos juegan un papel fundamental en la trama trófica marina como enlace clave entre el componente microbiano y los metazoos, todo ello debido a características como los cortos ciclos de vida con altas tasas de reproducción, herbivoría, etc. (Alder, 1999; Dolan, Ritchie, Tunin-Ley \& Pizay, 2009; Kamiyama \& Tsujino, 1996; Verity, 1985; Yu et al., 2015).

Diversos autores indican que el análisis de las comunidades planctónicas como los tintínidos es una herramienta para la construcción de cadenas tróficas que permitan entender el funcionamiento de un determinado hábitat (Cariou, Dolan \& Dallot, 1999; Li et al., 2015; Raybaud, Tunin-Ley, Ritchie \& Dolan, 2009; Santoferrara \& Alder, 2012; Villate, Uriarte, Olivar, Maynou, Emelianov \& Ameztoy, 2014; Wirtz, 2012). En su rol como agente conector y transfusor de materia y energía entre organismos de la base de las cadenas tróficas marinas, los tintínidos son considerados un importante consumidor herbívoro de detritus, picoplancton, bacterioplancton y de nanoplancton autotrófico y heterotrófico, así como de diatomeas y dinoflagelados (Alder, 1999; Hinder et al., 2012; Zhang, Zhang, Ni, Zhao, Huang \& Xiao, 2015). 
Lo anterior evidencia cómo el estudio de la población de tintínidos en las AMPR puede ser un punto importante en la compresión de la dinámica propia de un ecosistema, donde la productividad primaria es un punto medular para entender la producción secundaria que enriquece un hábitat determinado. El objetivo de este trabajo fue determinar la biodiversidad y abundancia de tintínidos y su relación con variables ambientales, en dos áreas marinas de pesca responsable del Golfo de Nicoya, Costa Rica, como punto de partida para entender la dinámica trófica de las AMPR.

\section{Materiales y métodos}

\section{Área de estudio}

Este estudio se realizó en dos áreas marinas de pesca responsable (AMPR) en el Golfo de Nicoya (GN), Costa Rica, específicamente en el AMPR-Puerto Níspero (PN) y en el AMPRPaquera (Paq), en los periodos de marzo del 2012 a marzo del 2013, y de setiembre de 2013 a agosto de 2014, respectivamente. El AMPR-Puerto Níspero se caracteriza por ubicarse en la parte interna del GN, donde hay una importante influencia de las aguas dulceacuícolas del río Tempisque y, además, tiene las aguas más someras del golfo. En el caso del área de Paquera, se define como una zona que presenta las mayores profundidades dentro del GN (25 a 100m) y por tener una fuerte influencia de las masas de agua oceánicas (Brenes \& León, 1995) (figura 1).

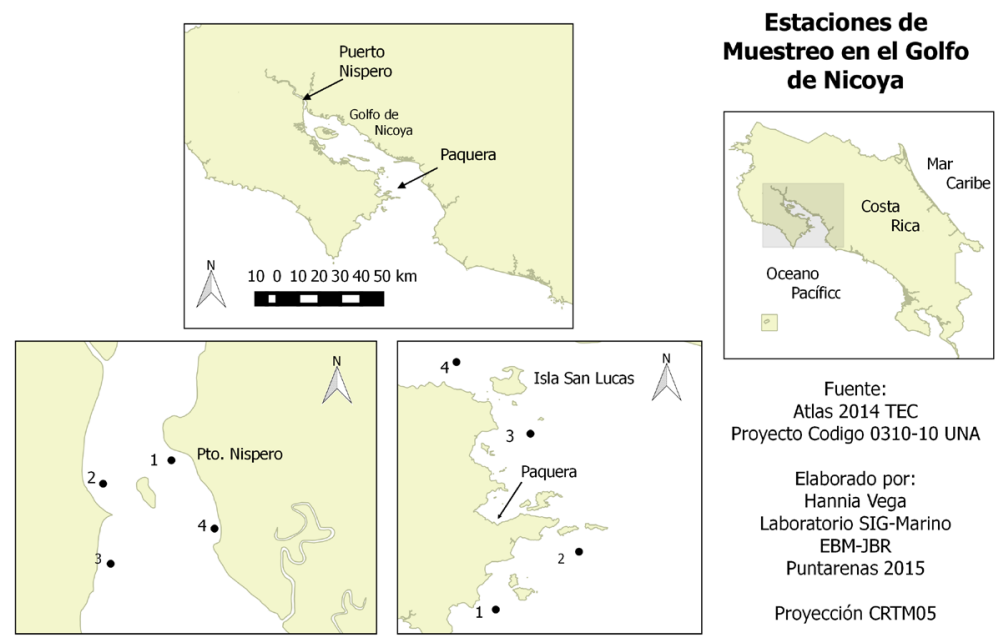

Figura 1. Ubicación de los puntos de muestreo en las AMPR Puerto Níspero y Paquera, Golfo de Nicoya, Costa Rica. Elaboración propia de la investigación.

\section{Variables ambientales y toma de muestras de agua}

Se seleccionaron cuatro estaciones de muestreo en el AMPR-Puerto Níspero y otras cuatro en el AMPR-Paquera, las cuales se muestrearon mensualmente durante el periodo de estudio en cada zona. En cada estación se tomaron muestras de agua para realizar análisis de la población de tintínidos y se midieron los parámetros físico-químicos con un multiparámetros Marca YSI 566. Las muestras de agua fueron recolectadas con una botella Niskin de cinco litros de 
UNICIENCIA Vol. 31, No. 1, pp. 1-12. Enero-junio, 2017.

capacidad, y a una profundidad de un metro en Níspero y a 2 metros en Paquera, y almacenadas en botellas de agua previamente etiquetadas. La diferencia en las profundidades se debió a que la zona de Puerto Níspero presenta en marea baja aguas muy someras. Para el análisis cualitativo y cuantitativo de los tintínidos se tomaron alícuotas de agua en botellas de $500 \mathrm{~mL}$ y se fijaron con una solución de Lugol para su conservación y posterior observación microscópica.

\section{Identificación y conteo de tintínidos}

El estudio de los tintínidos se realizó combinando la identificación taxonómica y la valoración cuantitativa de los organismos, mediante el método de sedimentación de Utermölh o con las celdas de conteo Segwich-Rafter. Para el análisis microscópico, se utilizó un microscopio de luz Nikon ECLIPSE E600, mediante el cual se realizó el recuento e identificación de los tintínidos observando todo el fondo de la abertura central de la cámara de sedimentación con un objetivo de 10x, 20x o 40x, dependiendo de las dimensiones de los organismos. La identificación se realizó hasta el taxón más bajo posible, mientras que los recuentos se ejecutaron agrupando las células de acuerdo con las especies o géneros identificados.

\section{Análisis de datos}

Para ambas AMPR, se elaboraron pruebas ANOVA de una vía para determinar si existían diferencias significativas temporales entre la abundancia de tintínidos y las variables ambientales; posteriormente, se comprobó la significancia de cada variable con una prueba de Posthoc de comparación múltiple de Student-Newman-Keuls (SNK). La prueba ANOVA se realizó con base en los datos recolectados en las cuatro estaciones de muestreo por el periodo de estudio y dentro de cada sitio.

La relación de la abundancia de tintínidos con respecto a los factores ambientales se determinó mediante una correlación de Pearson. Además, para determinar la relación entre la abundancia de tintínidos y su interacción con las variables ambientales en el tiempo, se realizó un análisis de correspondencias canónicas (ACC). Los análisis de ANOVA y correlación, así como el ACC se realizaron mediante el programa PAST versión 2.16 (Hammer, Harper \& Ryan, 2001).

\section{Resultados}

Las aguas del Golfo de Nicoya presentaron una marcada estacionalidad para ambas AMPR respecto a los parámetros físico-químicos, donde la dinámica propia del estuario influenciada por la magnitud de la descarga de agua dulce ya sea en época seca o lluviosa, determinó el comportamiento de las variables ambientales. Para el AMPR-PN todas las variables presentaron diferencias significativas entre los meses de muestreo $(A N O V A=0.001, p$-valor $<0.05)$, la temperatura, los sólidos totales, la salinidad y el oxígeno disuelto en la columna de agua, oscilaron entre valores de $27.3 \pm 0.2$ a $30.6 \pm 0.1{ }^{\circ} \mathrm{C}, 8.1 \pm 3.6$ a $23.0 \pm 1.6 \mathrm{mg} \mathrm{L}^{-1}, 7.3 \pm 3.5$ a $22.3 \pm 1.7$ ppm y $3.3 \pm 0.1$ a $6.0 \pm 0.1 \mathrm{mg} \mathrm{L}^{-1}$, respetivamente. La profundidad del disco Secchi fue un reflejo de la zonación interna donde se encuentra Puerto Níspero, al presentar valores en un rango de $0.09 \pm 0.04$ a $0.63 \pm 0.1 \mathrm{~m}$. La influencia marina se evidenció en las variables ambientales del AMPR-Paq, donde se observó una tendencia a menores temperatura $\left(27.0 \pm 1.2-30.0 \pm 0.1{ }^{\circ} \mathrm{C}\right)$, mayor concentración de sólidos totales (29.3 $\left.\pm 1.1-33.3 \pm 0.1 \mathrm{mg} \mathrm{L}^{-1}\right)$, salinidad (29.1 $\pm 1.2-33.5 \pm 0.1$ ppm) y oxígeno disuelto $\left(4.7 \pm 0.3-6.9 \pm 0.9 \mathrm{mg} \mathrm{L}^{-1}\right)$, y una mayor profundidad del disco Secchi (4.1 $\pm 3.3-9.3 \pm 5.5 \mathrm{~m})$ (tabla 1). Respecto al periodo de muestreo, a excepción de la profundidad 
ISSN Electrónico: 2215-3470

DOI: http://dx.doi.org/10.15359/ru.31-1.
UNICIENCIA Vol. 31, No. 1, pp. 1-12. Enero-junio, 2017. URL: www.revistas.una.ac.cr/uniciencia Email: revistauniciencia@una.cr

del disco Secchi $(\mathrm{ANOVA}=0.949$, $\mathrm{p}$-valor $>0.05)$, las demás variables presentaron diferencias significativas temporales (ANOVA $=0.001, \mathrm{p}$-valor $<0.05)$.

Tabla 1

Valores promedio de las variables ambientales en las AMPR Puerto Níspero y Paquera, en el Golfo de Nicoya, Costa Rica

\begin{tabular}{cccccc}
\hline \multicolumn{5}{c}{ AMPR-Puerto Níspero } \\
\hline Mes & $\mathrm{T}\left({ }^{\circ} \mathrm{C}\right)$ & ST $\left(\mathrm{mg} \mathrm{L}^{-1}\right)$ & Sal $(\mathrm{ppm})$ & $\mathrm{O}_{2}\left(\mathrm{mg} \mathrm{L}^{-1}\right)$ & $\mathrm{DS}(\mathrm{m})$ \\
\hline Mar-12 & $28.9 \pm 0.3$ & $23.0 \pm 1.6$ & $22.3 \pm 1.7$ & $5.9 \pm 0.5$ & $0.31 \pm 0.03$ \\
Abr-12 & $28.8 \pm 0.4$ & $20.3 \pm 3.9$ & $20.0 \pm 4.3$ & $5.6 \pm 0.3$ & $0.38 \pm 0.1$ \\
May-12 & $29.8 \pm 0.3$ & $16.8 \pm 5.0$ & $15.8 \pm 5.0$ & $4.9 \pm 0.3$ & $0.61 \pm 0.05$ \\
Jun-12 & $28.7 \pm 0.1$ & $14.9 \pm 0.7$ & $13.7 \pm 0.9$ & $5.0 \pm 0.4$ & $0.56 \pm 0.1$ \\
Jul-12 & $28.9 \pm 1.1$ & $11.9 \pm 2.0$ & $11.4 \pm 2.1$ & $5.0 \pm 0.2$ & $0.23 \pm 0.05$ \\
Ago-12 & $29.6 \pm 0.2$ & $12.2 \pm 3.0$ & $10.8 \pm 2.7$ & $4.9 \pm 0.3$ & $0.63 \pm 0.1$ \\
Set-12 & $30.6 \pm 0.1$ & $20.7 \pm 2.0$ & $19.7 \pm 2.1$ & $3.3 \pm 0.1$ & $0.44 \pm 0.05$ \\
Nov-12 & $27.3 \pm 0.2$ & $8.1 \pm 3.6$ & $7.3 \pm 3.5$ & $5.1 \pm 0.1$ & $0.88 \pm 0.05$ \\
Dic-12 & $27.7 \pm 0.2$ & $23.0 \pm 1.6$ & $22.3 \pm 1.7$ & $6.0 \pm 0.1$ & $0.24 \pm 0.1$ \\
Ene-13 & $28.6 \pm 0.4$ & $17.7 \pm 1.9$ & $16.6 \pm 2.0$ & $4.7 \pm 0.6$ & $0.09 \pm 0.04$ \\
Feb-13 & $29.1 \pm 0.1$ & $20.3 \pm 1.4$ & $19.4 \pm 1.6$ & $5.4 \pm 0.4$ & $0.16 \pm 0.03$ \\
Mar-13 & $29.6 \pm 0.2$ & $21.7 \pm 4.1$ & $20.2 \pm 3.4$ & $5.9 \pm 0.6$ & $0.51 \pm 0.03$ \\
\hline & & AMPR-Paquera & & \\
\hline Mes & $\mathrm{T}\left({ }^{\circ} \mathrm{C}\right)$ & $\mathrm{ST}\left(\mathrm{mg} \mathrm{L} \mathrm{L}^{-1}\right)$ & Sal $(\mathrm{ppm})$ & $\mathrm{O}_{2}\left(\mathrm{mg} \mathrm{L}^{-1}\right)$ & $\mathrm{DS}(\mathrm{m})$ \\
Set-13 & $27.2 \pm 0.3$ & $30.7 \pm 0.8$ & $30.7 \pm 0.9$ & $5.2 \pm 0.3$ & $4.6 \pm 3.5$ \\
Oct-13 & $27.6 \pm 0.4$ & $29.3 \pm 1.1$ & $29.1 \pm 1.2$ & $5.7 \pm 1.0$ & $4.9 \pm 3.0$ \\
Nov-13 & $27.6 \pm 0.6$ & $30.0 \pm 0.9$ & $29.9 \pm 1.0$ & $4.7 \pm 0.3$ & $4.1 \pm 3.3$ \\
Dic-13 & $27.7 \pm 0.5$ & $30.6 \pm 0.1$ & $30.5 \pm 0.1$ & $5.9 \pm 0.8$ & $6.6 \pm 3.8$ \\
Ene-13 & $27.8 \pm 0.6$ & $31.7 \pm 0.3$ & $31.8 \pm 0.4$ & $5.7 \pm 0.5$ & $6.2 \pm 5.9$ \\
Feb-14 & $27.0 \pm 1.2$ & $32.5 \pm 0.2$ & $32.7 \pm 0.2$ & $6.1 \pm 0.4$ & $7.9 \pm 5.1$ \\
Mar-14 & $27.6 \pm 0.5$ & $33.0 \pm 0.2$ & $33.2 \pm 0.3$ & $6.4 \pm 0.6$ & $5.0 \pm 4.4$ \\
Abr-14 & $30.0 \pm 0.1$ & $33.3 \pm 0.1$ & $33.5 \pm 0.1$ & $5.6 \pm 0.0$ & $6.7 \pm 6.1$ \\
May-14 & $29.7 \pm 0.1$ & $32.3 \pm 0.3$ & $32.3 \pm 0.3$ & $6.9 \pm 0.9$ & $8.0 \pm 4.3$ \\
Jun-14 & $28.9 \pm 0.1$ & $32.1 \pm 0.1$ & $32.2 \pm 0.1$ & $6.1 \pm 0.7$ & $6.4 \pm 6.6$ \\
Jul-14 & $29.8 \pm 0.4$ & $31.9 \pm 0.1$ & $31.9 \pm 0.1$ & $6.6 \pm 0.2$ & $9.3 \pm 5.5$ \\
Ago-14 & $29.1 \pm 0.2$ & $31.5 \pm 0.1$ & $31.5 \pm 0.1$ & $6.2 \pm 0.0$ & $6.8 \pm 5.4$ \\
\hline & & & &
\end{tabular}

Nota: $(\mathrm{T}=$ Temperatura; $\mathrm{ST}=$ Sólidos totales; $\mathrm{Sal}=$ Salinidad; $\mathrm{O} 2=$ Oxígeno disuelto; $\mathrm{DS}=$ Profundidad disco Secchi). Elaboración propia de la investigación.

Sobre la población de tintínidos, se evidenció una baja riqueza específica de especies para ambos sitios de muestreo, así como una tendencia a que la mayoría de organismos identificados se presentaron de manera esporádica en las muestras analizadas. Para el AMPR-PN se identificaron 
UNICIENCIA Vol. 31, No. 1, pp. 1-12. Enero-junio, 2017.

Email: revistauniciencia@una.cr

16 especies, mientras que para el AMPR-Paq ocho especies. Las especies más frecuentemente observadas son Tintinnopsis parva para ambos sitios, además, Tintinnidium balechi y Tintinnopsis uruguayensis para la zona de Puerto Níspero (tabla 2). Al igual que la riqueza, la abundancia de los ciliados fue mayor en el AMPR-PN con concentraciones de tintínidos que oscilaron entre 100 y 5583 cél $\mathrm{L}^{-1}$, presentó la mayor abundancia en el mes de setiembre del 2012 y una diferenciación significativa entre los meses de muestreo (ANOVA $=0.001$, $\mathrm{p}$-valor $<0.05$ ); mientras en el AMPR-

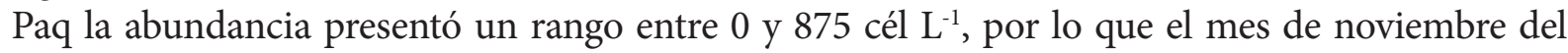
2013 fue el mes de mayor abundancia de tintínidos, sin embargo, en esta zona no se evidenciaron diferencias significativas entre el periodo de muestreo (ANOVA $=0.443$, p-valor $>0.05)$ (figura 2).

Tabla 2

Riqueza de tintínidos en cada periodo de muestreo para las AMPR Puerto Níspero y Paquera, en el Golfo de Nicoya, Costa Rica.

\begin{tabular}{|c|c|c|c|c|c|c|c|c|c|c|c|c|}
\hline AMPR-Puerto Níspero & $\begin{array}{c}\text { mar- } \\
12 \\
\end{array}$ & $\begin{array}{c}\text { abr- } \\
12\end{array}$ & $\begin{array}{c}\text { may- } \\
12\end{array}$ & $\begin{array}{c}\text { jun- } \\
12\end{array}$ & $\begin{array}{c}\text { jul- } \\
12\end{array}$ & $\begin{array}{c}\text { ago- } \\
12\end{array}$ & $\begin{array}{c}\text { set- } \\
12\end{array}$ & $\begin{array}{c}\text { nov- } \\
12\end{array}$ & $\begin{array}{c}\text { dic- } \\
12\end{array}$ & $\begin{array}{c}\text { ene- } \\
13\end{array}$ & $\begin{array}{c}\text { feb- } \\
13\end{array}$ & $\begin{array}{c}\text { mar- } \\
13\end{array}$ \\
\hline Codonella aspera & + & & & & & & & & & & & \\
\hline Codonella pusilla & & + & & & & & & & & & & \\
\hline Codonella sp & & & + & & & & & & & & & \\
\hline Codonellopsis morchella & & + & & & & & & & & & & \\
\hline Eutintinnus fraknoi & & & & & & & & & + & & & \\
\hline Favella taraikaensis & & & + & & & & & & & & & \\
\hline Protorhabdonella simplex $c f$. & & & + & & & & & & & & & \\
\hline Tintinnidium balechi & & + & + & & + & + & + & + & & + & & \\
\hline Tintinnidium sp. aff. T. semiciliatum & + & + & & & & & & & & & & \\
\hline Tintinninopsis lobiancoi & & + & + & + & & & & + & & & & \\
\hline Tintinnopsis parva & & + & + & + & + & + & + & + & + & + & & \\
\hline Tintinninopsis radix & + & + & & & & & & & & & & \\
\hline Tintinnopsis tocantinensis & + & & & & & & & & & & & \\
\hline Tintinnopsis uruguayensis & & + & + & + & + & & + & + & & + & & \\
\hline Undella claperei & + & & & + & + & & & & & & & \\
\hline Undella hyalina & & & & & & & & + & & & & \\
\hline AMPR-Paquera Tambor & $\begin{array}{c}\text { set- } \\
13\end{array}$ & $\begin{array}{c}\text { oct- } \\
13\end{array}$ & $\begin{array}{c}\text { nov- } \\
13\end{array}$ & $\begin{array}{c}\text { dic- } \\
13\end{array}$ & $\begin{array}{c}\text { ene- } \\
14\end{array}$ & $\begin{array}{c}\text { feb- } \\
14\end{array}$ & $\begin{array}{c}\text { mar- } \\
14\end{array}$ & $\begin{array}{c}\text { abr- } \\
14\end{array}$ & $\begin{array}{c}\text { may- } \\
14\end{array}$ & $\begin{array}{c}\text { jun- } \\
14\end{array}$ & $\begin{array}{c}\text { jul- } \\
14\end{array}$ & $\begin{array}{c}\text { ago- } \\
14\end{array}$ \\
\hline Codonellopsis gaussi cf. Coxliella & & & + & & & & & & & & & \\
\hline Eutintinnus fracknoi & + & & & & & & & & & & & \\
\hline Salpingella $c f$. & & + & & & & & & + & & & & \\
\hline Tintinnopsis lobiancoi & + & & & & & & & & & & & \\
\hline Tintinnopsis fimbriata & + & & + & & & & & & & & & \\
\hline Tintinnopsis parva & & + & + & + & + & & + & & + & & & + \\
\hline Tintinnopsis radix & & & + & & & & & & & & & \\
\hline Undella claparedei & & & + & & & & & & & & & \\
\hline
\end{tabular}

Nota: Elaboración propia de la investigación. 
Al correlacionar la abundancia de tintínidos con respecto a las variables ambientales estudiadas en cada sitio de muestreo, se determinó una tendencia a que las interacciones significativas fueran negativas. Para la zona de Puerto Níspero, únicamente se presentó una correlación negativa significativa entre la abundancia de tintínidos y la concentración de oxígeno disuelto. Esta misma relación se presentó en el área de Paquera, donde además se vio una tendencia a que los tintínidos tuvieran una correlación negativa significativa respecto a la salinidad, los sólidos totales y la profundidad del disco Secchi (tabla 3).
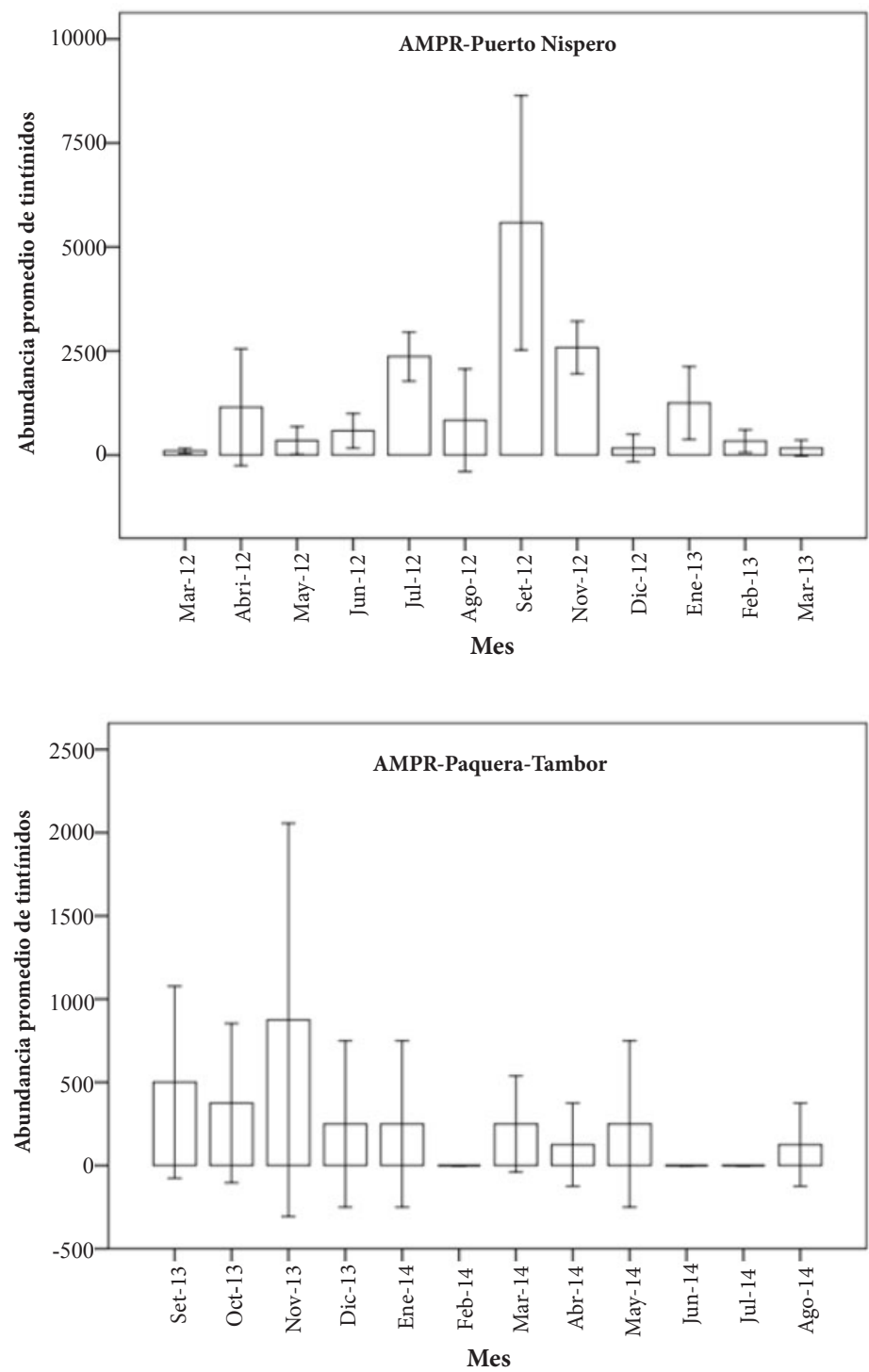

Figura 2. Abundancia promedio de tintínidos (cél $\left.\mathrm{L}^{-1}\right)$ en las AMPR Puerto Níspero y Paquera, en el Golfo de Nicoya, Costa Rica (las barras son indicativas de la desviación estándar). Elaboración propia de la investigación. 
UNICIENCIA Vol. 31, No. 1, pp. 1-12. Enero-junio, 2017.

Tabla 3

Correlaciones de Pearson significativas a un p-valor $<0.05$ entre la abundancia de tintínidos y las variables ambientales en las AMPR Puerto Níspero y Paquera, en el Golfo de Nicoya, Costa Rica.

\begin{tabular}{ccc}
\hline & AMPR-Níspero & AMPR-Paquera \\
\hline Tin- ${ }_{2}$ & -0.68 & -0.33 \\
Tin-ST & - & -0.33 \\
Tin-Sal & - & -0.32 \\
Tin-DS & - & -0.34 \\
\hline
\end{tabular}

Nota: Elaboración propia de la investigación.

Al interrelacionar de qué manera el total de las variables ambientales influyó en el comportamiento temporal de la abundancia de tintínidos, se determinó que los parámetros físico-químicos tienen una proyección relevante principalmente en los meses de mayor abundancia de los organismos ciliados estudiados para ambas zonas, es decir, en setiembre del 2012 en el AMPR-PN, y en noviembre del 2013 en el AMPR-Paq (figura 3).
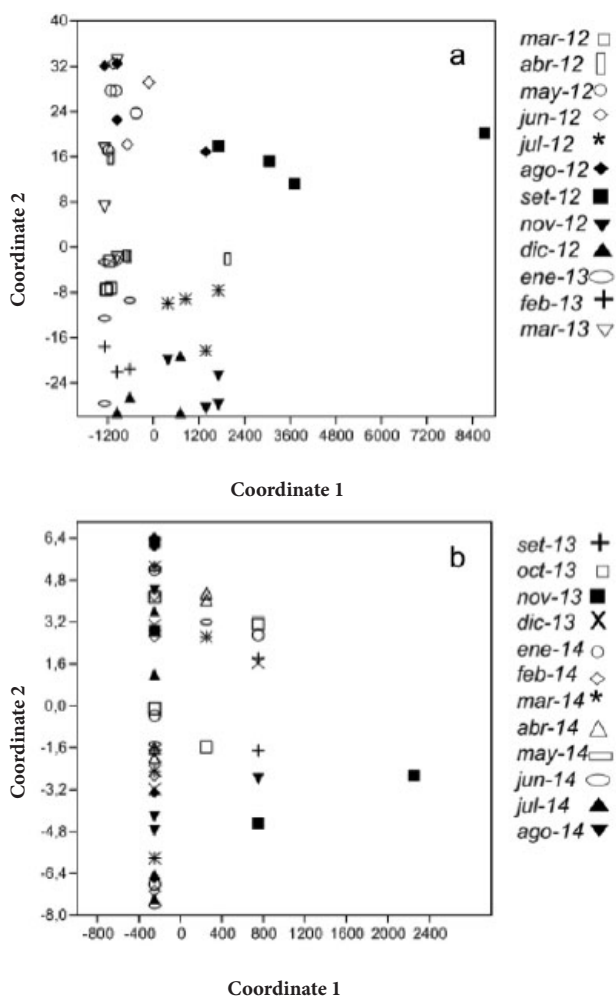

Figura 3. Análisis de Correspondencias Canónicas entre la abundancia de tintínidos y las variables ambientales según los meses de muestreo en las AMPR Puerto Níspero (a) y Paquera (b), en el Golfo de Nicoya, Costa Rica. Elaboración propia de la investigación. 
ISSN Electrónico: 2215-3470

DOI: http://dx.doi.org/10.15359/ru.31-1.1
UNICIENCIA Vol. 31, No. 1, pp. 1-12. Enero-junio, 2017. URL: www.revistas.una.ac.cr/uniciencia Email: revistauniciencia@una.cr

\section{Discusión}

La variación de los factores ambientales en los periodos de muestreo reflejó la importancia de los cambios estacionales sobre las propiedades físico-químicas del agua del Golfo de Nicoya, tanto en la zona interna del golfo donde se ubica el AMPR-PN como en la parte externa donde está el AMPR-Paq. La disminución gradual de la salinidad, la temperatura desde inicios de la época lluviosa y la subsecuente elevación de estos parámetros conforme inicia la época seca podrían explicarse principalmente por los efectos de las precipitaciones asociadas a la época lluviosa (Solano-León, 2012), donde se da un incremento en el caudal del río Tempisque, el Barranca y el Grande de Tárcoles. Estos ríos tienen influencia directa en las zonas de estudio, aumentan el aporte de agua dulce y disminuyen la concentración de sal y temperatura del agua, además de la concentración de oxígeno en el agua (Brenes, León \& Chávez, 2001; Brenes, 2001; Epifanio, Maurer \& Dittel, 1983; Kress, León, Brenes, Brenner \& Arroyo, 2001; Lizano \& Vargas 1994; Voorhis, Epifanio, Maurer, Dittel \& Vargas, 1983).

Al comparar ambas AMPR respecto a las variables ambientales se evidencia una zonación del Golfo de Nicoya de acuerdo con las características de la zona interna y externa del golfo, donde las condiciones internas de aguas someras y la entrada directa de las aguas del río Tempisque hacen que las temperaturas aumenten y la salinidad, sólidos totales, concentración de oxígeno disuelto y profundidad del disco Secchi disminuyan; caso contrario en la zona externa donde hay mayores profundidades y un efecto directo de las aguas oceánicas (Brenes y León, 1995). Esta zonación también aplica para la abundancia y diversidad de los tintínidos, ya que la zona interna de Puerto Níspero presentó condiciones más idóneas para mantener una población de estos organismos más diversa y con una mayor concentración, que la zona de Paquera. Los tintínidos son uno de los grupos de ciliados marinos que sirven como indicador para la diferenciación de masas de agua (Feng, Zhang, Wang, Zhang, Xiao \& Xu, 2015; Zhang et al., 2015). Además, la movilidad limitada de los ciliados hace que la distribución y agregación de estos organismos esté fuertemente influenciada por factores físico-químicos como corrientes y agua dulce (Li et al.; 2015; Moscatello et al., 2011; Rakshit et al., 2015a; Reid \& Stewart, 1989; Sitran, Bergamasco, Decembrini \& Guglielmo, 2007).

La abundancia de tintínidos en el Golfo de Nicoya se puede considerar baja al compararla con sitios tanto tropicales como templados. En el estuario del río Hooghly en la India, se determinó la estructura comunitaria de tintínidos durante un periodo anual, con concentraciones que oscilaron entre 5097 \pm 307 y $9113 \pm 368$ individuos $\mathrm{L}^{-1}$. Asimismo, en el estuario de Bahía Blanca, Argentina, se determinó durante un ciclo anual la distribución estacional de tintínidos y su relación con las variables ambientales, contabilizaron abundancias de estos ciliados entre 20 y 12400 individuos $\mathrm{L}^{-1}$. Para ambos sitios, las concentraciones fueron muy superiores a las encontradas en las AMPR del Golfo de Nicoya.

Las especies frecuentes Tintinnopsis parva, T. uruguayensis y Tintinnidium balechi pertenecen a grupos de tintínidos que se han caracterizado como organismos dominantes en zonas costeras y con influencia dulceacuícola (Elliot \& Kaufmann, 2007; Trottet, Roy, Tamigneaux \& Lovejoy, 2007), y en el caso del género Tintinnopsis se considera un grupo de especies típicamente costeras (ذiang, Yang, Al-Farraj, Warren \& Lin, 2012; Sitran et al., 2007). Para algunos autores, la presencia de tintínidos es el reflejo de un sistema donde la producción primaria juega un papel fundamental en el desarrollo de la estructura trófica, ya que, según Gómez (1997), el desarrollo de tintínidos coincide con el florecimiento del fitoplancton, y juega un papel muy importante 
UNICIENCIA Vol. 31, No. 1, pp. 1-12. Enero-junio, 2017.

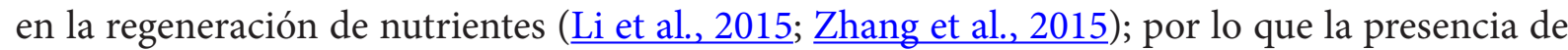
tintínidos es un posible indicador de que la zona exhibe un entorno nutricionalmente rico y de gran productividad primaria.

En este trabajo, la correlación entre la abundancia de tintínidos y la concentración de oxígeno fue significativamente negativa, lo cual se evidencia en los muestreos de mayor abundancia de tintínidos tanto en el AMPR-PN como en el AMPR-Paq, que es también donde se dan los valores menores de oxígeno disuelto. Según Zhang et al. (2015), en el estuario de Changjiang, Mar de China, la abundancia y biomasa de ciliados como los tintínidos es común en zonas donde la disminución del oxígeno en las aguas es evidente producto de procesos de hipoxia temporales. Mencionan, además, que especies como Tintinnopsis parva y especies del género Tintinnidium pueden llegar a representar entre el 41-99\% de la biomasa total en zonas con fondos anóxicos. Si bien en las zonas de estudio no se determinaron las variables ambientales del bentos, el Golfo de Nicoya se caracteriza, principalmente en la zona interna, por la presencia de bosques de manglar, que le confiere una alta carga de materia orgánica a la columna de agua y al sedimento, lo que puede originar una mayor demanda de oxígeno y, con ello, una disminución en la concentración de este gas en la zona.

La presencia de tintínidos en las AMPR de Puerto Níspero y Paquera evidencia una estructura trófica dinámica, donde las condiciones propias de cada zona enriquecen y regulan la productividad. Según Suthers y Rissik (2009), la circulación del agua y los gradientes de salinidad son las principales fuerzas físicas que influyen en la dinámica de poblaciones de zooplancton, además de los procesos de mezclado, los cuales también afectan la productividad de los estuarios, ya que las mareas o descarga de los ríos, a menudo, introducen nutrientes y la mezcla producida por el viento puede volver a suspender las partículas de materia orgánica a lo largo de los márgenes de los estuarios y ofrecer una mayor oferta de alimentos a filtradores bentónicos y planctónicos, promoviendo así el intercambio de nutrientes entre los sedimentos y la columna de agua.

\section{Agradecimientos}

A las Asociaciones de Pescadores de las AMPR Puerto Níspero y Paquera por la colaboración y disposición para la realización de los muestreos en las áreas que ellos administran. Al proyecto de la Escuela de Ciencias Biológicas "Identificación y evaluación de los hábitats como zonas de pesca responsable en el litoral del Pacífico Central de Costa Rica” código SIA-0310-10, por el apoyo y financiamiento para el trabajo realizado.

\section{Referencias}

Abou Zaid, M. M. \& Hellal, A. M. (2012). Tintinnids (Protozoa: Ciliata) from the coast of Hurghada Red Sea, Egypt. Egyptian Journal of Aquatic Research, 38, 249-268. http://dx.doi.org/10.1016/j. ejar.2013.01.003

Alder, V. A. (1999). Tintinnoidea. En D- Boltovskoy, (Ed.), South Atlantic Zooplankton, (Vol. II, pp. 321384). Leiden, The Nerthelands: Backhuys Publishers.

Brenes, C. \& León, S. (1995). Hidrografía del Golfo de Nicoya, Costa Rica. Ponencia presentada en el Simposium Ecosistemas de Manglares en el Pacífico Centroamericano. San Salvador, El Salvador.

Brenes C. (2001). Fundamentos de oceanografía descriptiva: Aplicación al istmo centroamericano. Nicaragua: Proyecto para el Desarrollo Integral de la Pesca Artesanal en la Región Autónoma Atlántico Sur (DIPAL).

Brenes, C. L., Leon, S., \& Chávez, J. (2001). Variación de las propiedades termohalinas en el Golfo de Nicoya, Costa Rica. Revista de Biología Tropical, 49, 145-152. 
ISSN Electrónico: 2215-3470

DOI: http://dx.doi.org/10.15359/ru.31-1.1
UNICIENCIA Vol. 31, No. 1, pp. 1-12. Enero-junio, 2017.

URL: www.revistas.una.ac.cr/uniciencia Email: revistauniciencia@una.cr

Cariou, J. B., Dolan, J. R. \& Dallot, S. (1999). A preliminary study of tintinnid diversiy in the NW Mediterranean Sea. Journal of Plankton Research, 21(6), 1065-1075. http://dx.doi.org/10.1093/ plankt/21.6.1065.

Dolan, J. R., Ritchie, M. E., Tunin-Ley, A. \& Pizay, M. D. (2009). Dynamics of core and occasional species in the marine plankton: Tintinnid ciliates in the north-west Mediterranean Sea. Journal of Biogeography, 36, 887-895. http://dx.doi.org/10.1111/j.1365-2699.2008.02046.x

Elliot, D. T. \& Kaufmann, R. S. (2007). Spatial and Temporal Variability of Mesozooplankton and Tintinnids Ciliates in a Seasonally Hypersaline Estuary. Estuaries and Coasts, 30(3), 418-430. http://dx.doi. org/10.1007/BF02819388

Epifanio, C. E., Maurer, D. \& Dittel, A. I. (1983). Seasonal changes in nutrients and dissolved oxygen in the Gulf of Nicoya, a tropical estuary on the Pacific coast of Central America. Hydrobiologia, 101, 231-238. http://dx.doi.org/10.1007/BF00009879

Feng, M., Zhang, W., Wang, W., Zhang, G., Xiao, T. \& Xu, H. (2015). Can tintinnids be used for discriminating water quality status in marine ecosystems? Marine Pollution Bulletin. http://dx.doi. org/10.1016/j.marpolbul.2015.10.059

Gómez, O. (1997). Los tintínidos (Protozoa: Ciliata) del mar peruano y sus relaciones con el fenómeno El Niño. Boletín del Instituto del Mar de Perú, 16(2), 61-84.

Hammer, Ø.; Harper, D. A. T. \& Ryan, P. D. (2001). PAST: Paleontological Statistics software package for education and data analysis. Paleontologia Electronica 4(1), 9.

Hinder, S. L., Manning J. E., Gravenor, M. B., Edwards, M., Walne, A. W., Burkill, P. H. \& Hays, G. C. (2012). Long-term chages in abundance and distribution of microzooplankton in the NE Atlantic and North Sea. Journal of Plankton Research, 34(1), 83-91. http://dx.doi.org/10.1093/plankt/fbr087

Jiang, Y., Yang, J., Al-Farraj, S. A., Warren, A. \& Lin, X. (2012). Redescriptions of three tintinnids ciliates, Tintinnopsis tocantinensis, T. radix and T. cylindrical (Ciliophora, Spirotrichea), from coastal waters off China. European Journal of Protistology, 48, 314-325. http://dx.doi.org/10.1016/j.ejop.2012.02.001

Kamiyama, T. \& Tsujino, M. (1996). Seasonal variation in the species composition of tintinnid ciliates in Hiroshima Bay, the Seto Inland Sea of Japan. Journal of Plankton Research, 18(12), 2313-2327. http:// dx.doi.org/10.1093/plankt/18.12.2313

Kress, N., León, S., Brenes, C., Brenner, S. \& Arroyo, G. (2001). Horizontal transport and seasonal distribution of nutrients, dissolved oxygen and chlorophyll-a in the Gulf of Nicoya, Costa Rica: a tropical estuary. Continental Shelf Research, 22(1), 51-66. http://dx.doi.org/10.1016/S0278-4343(01)00064-4

Li, H., Zhao, Y., Chen, X., Zhang, W., Xu, J., Li, J. \& Xiao, T. (2015). Interaction between neritic and warm water tintinnids in surface waters of East China Sea. Deep-Sea Research II, http://dx.doi. org/10.1016/j.dsr2.2015.06.008

Lizano, O. \& Vargas, J. (1994). Distribución espacio-temporal de la salinidad y la temperatura en la parte interna del Golfo de Nicoya. Tecnología en Marcha, 12(2), 3-16.

Moscatello, S., Caroppo, C., Hajdëri, E. \& Belmonte, G. (2011). Space Distribution of Phyto- and Microzooplankton in the Vlora Bay (Southern Albania, Mediterranean Sea). Journal of Coastal Research, 58, 80-94. http://dx.doi.org/10.2112/SI $58 \quad 8$

Rakshit, D., Kumar Sarkar, S., Deb Bhattacharya, B., Jonathan, M. P., Kumar Biswas, J., Mondal, P. \& Mitra, S. (2015a). Human-induced ecological changes in western part of Indian Sundarban megadelta: A threat to ecosystem stability. Marine Pollution Bulletin, 99, 186-194. http://dx.doi.org/10.1016/j. marpolbul.2015.07.027

Rakshit, D., Sankar Ganesh, P. \& Kumar Sarkar, S. (2015b). Choreotrich ciliate tintinnid (Protozoa: Ciliophora) in a tropical meso-macrotidal estuary, eastern part of India. Regional Studies in Marine Science, http://dx.doi.org/10.1016/j.rsma.2015.06.003 
UNICIENCIA Vol. 31, No. 1, pp. 1-12. Enero-junio, 2017.

ISSN Electrónico: 2215-3470

URL: www.revistas.una.ac.cr/uniciencia

DOI: http://dx.doi.org/10.15359/ru.31-1.1

Raybaud, V., Tunin-Ley, A., Ritchie, M. E. \& Dolan, J. R. (2009). Similar patterns of community organization characterize distinct groups of different trophic levels in the plankton of the NW Mediterranean Sea. Biogeosciences, 6, 431-438. http://dx.doi.org/10.5194/bg-6-431-2009

Reid, F. M. H. \& Stewart, E. (1989). Nearshore microplaktonic assemblages off southern California in Febrary 1983 during the El Niño event. Continental Shelf Research, 9, 37-50. http://dx.doi. org/10.1016/0278-4343(89)90081-2

Santoferrara, L. \& Alder, V. (2012). Abundance and diversity of tintinnids (planktonic ciliates) under contrasting levels of productivity in the Argentine Shelf and Drake Passage. Journal of Sea Research, 71, 25-30. http://dx.doi.org/10.1016/j.seares.2012.04.002

Sitran, R., Bergamasco, A., Decembrini, F. \& Guglielmo, L. (2007). Temporal succession of tintinnids in the northern Ionian Sea, Central Mediterranean. Journal of Plankton Research, 29(6), 495-508. http://dx.doi.org/10.1093/plankt/fbm032

Solano-León, E. (2012). Resumen meteorológico diciembre de 2012. Boletín Meteorológico Mensual. Departamento de Meteorología Sinóptica y Aeronáutica (DMSA) Instituto Meteorológico Nacional, Costa Rica. Recuperado de https://www.imn.ac.cr/documents/10179/14635/DICIEMBRE

Suthers, I. \& Rissik, D. (2009). Plankton: a guide to their ecology and monitoring for water quality. Collingwood, Victoria, Australia: CSIRO Publishing.

Thompson, G. A., Alder, V. A. \& Boltovskoy, D. (2001). Tintinnids (Ciliophora) and Other Net Microzooplankton $(>30 \mu \mathrm{m})$ in South-western Atlantic Shelf Break Waters. Marine Ecology, 22(4), 343355. http://dx.doi.org/10.1046/j.1439-0485.2001.01723.x

Trottet, A., Roy, S., Tamigneaux, E. \& Lovejoy, C. (2007). Importance of heterotrophic planktonic communities in a mussel culture environment: Grande Entrée laggon, Magdalen Islands (Québec, Canada). Marine Biology, 151, 377-392. http://dx.doi.org/10.1007/s00227-006-0494-y

Verity, P. (1985). Grazing, repiration, excretion, and growth rates of tintinnids. Limnology and Oceanography, 30(6), 1268-1282. http://dx.doi.org/10.4319/lo.1985.30.6.1268

Villate, F., Uriarte, I., Pilar Olivar, M., Maynou, F., Emelianov, M. \& Ameztoy, I. (2014). Mesoscale structure of microplankton and mesoplankton assemblages under contrasting oceanographic conditions in the Catalan Sea (NW Mediterranean). Journal of Marine Systems, 139, 9-26. http://dx.doi. org/10.1016/j.jmarsys.2014.05.004

Voorhis, A., Epifanio, C., Maurer, D., Dittel, A. \& Vargas, J. (1983). The estuarine character of the Gulf of Nicoya, an embayment on the Pacific coast of de Central America. Hydrobiologia, 99, 225-237. http://dx.doi.org/10.1007/BF00008774

Wirtz, K.W. (2012). Who is eating whom? Morphology and feeding habits of co-occurring sprat (Sprattus sprattus) and cod (Gabus morhua) larvae in the Bornholm Basin, Baltic Sea. Fisheries Research, 63, 97-111.

Yu, Y., Zhang, W., Feng, M., Zhao, Y., Zhang, C., Zhou, F. \& Xiao, T. (2015). Differences in the vertical distribution and response to freshwater discharge between aloricate ciliates and tintinnids in the East China Sea. Journal of Marine Systems, http://dx.doi.org/10.1016/j.jmarsys.2015.02.005

Zhang, C., Zhang, W., Ni, X., Zhao, Y., Huang, L. \& Xiao, T. (2015). Influence of different wáter masses on planktonic ciliate distribution on the East China Sea self. Journal of Marine Systems, 141, 98-11. http://dx.doi.org/10.1016/j.jmarsys.2014.09.003

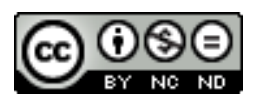

Dinámica anual de tintínidos en las aguas estuarinas de dos Áreas Marinas de Pesca Responsable en el Golfo de Nicoya, Costa Rica (Andrea García-Rojas y otros) por Revista Uniciencia se encuentra bajo una Licencia CreativeCommons Atribución-NoComercial-SinDerivadas 3.0 Unported. 